Applied Mathematical Sciences Vol. 8, 2014, no. 133, 6709 - 6716

HIKARI Ltd, www.m-hikari.com

http://dx.doi.org/10.12988/ams.2014.49685

\title{
An Interdisciplinary Laboratory in Mathematics and Music
}

\author{
Salvatore Cuomo*, Ardelio Galletti ${ }^{+}$, Gabriele Guerriero* \\ *Dipartimento di Matematica e Applicazioni "R. Caccioppoli" \\ Università degli Studi di Napoli "Federico II" \\ Via Cintia, Complesso Monte S. Angelo, 80126 Naples, Italy \\ ${ }^{+}$Dipartimento di Scienze e Tecnologie \\ Università degli Studi di Napoli "Parthenope" \\ Centro Direzionale, Isola C4, 80143 Naples, Italy
}

Copyright (C) 2014 Salvatore Cuomo, Ardelio Galletti and Gabriele Guerriero. This is an open access article distributed under the Creative Commons Attribution License, which permits unrestricted use, distribution, and reproduction in any medium, provided the original work is properly cited.

\begin{abstract}
This paper concerns the connection between Mathematics and Music. After a description of the nature of sound, the problem of the construction of the musical scale, from the studies of Pythagoras to the tempered scale is considered. An algorithm for the construction of a scale is given.
\end{abstract}

Mathematics Subject Classification: 97Q80, 97N80, 97N10

Keywords: Musical scale, algorithm, Pythagoras scale

\section{Introduction}

Some year ago we participated in a lab entitled "Models for the Community". In order to show the strict connection between Mathematics and Physics, we decided to use a subject that is really interesting for young students: music. We considered several aspects starting from the nature of sound and its propagation in waves. This one is the part mostly connected to Physics. After we treated the musical scale from the studies of Pythagoras to the tempered scale 
of nowadays. Due to the age of the students we did not use the Fourier series but just the properties connected to trigonometric functions, already known by high school students. Hence, in this paper, firstly we recall our past teaching experiences and finally we give a short algorithm for the construction of a temperate musical scale. The paper is organized as follows: in Section 2 we consider sound waves from a physical point of view; in Section 3 we deal with the problem of a musical scale construction and we present an algorithm for doing it; finally, in Section 4 we draw conclusions.

\section{Riding the sound waves}

Sound is a vibratory motion of the air or any other media, it does not travel in a vacuum. The sound is determined by vibrating bodies and transmitted to our ears without sensible motions of the air. At this point our ears start to vibrate, and this vibration is interpreted by the brain as sound. Clearly vibratory phenomena have waveform. We may say that sound waves travel through air almost in the same way as water waves travel through water. An acoustic resonator is a device that exhibits resonance or resonant behavior, that is, it naturally oscillates at some frequencies, called its resonant frequencies, with greater amplitude than at others. It is used in order to enhance the effect of the vibrations of the air and make the sound more clear. The oscillations in an acoustic resonator are mechanical. Resonators are used to either generate waves of specific frequencies or to select specific frequencies from a signal. Musical instruments use acoustic resonators that produce sound waves of specific tones. A sound wave has the same characteristics as any other type of waveform: wavelength, frequency, and amplitude. The wavelength is the distance from one crest to another of a wave. The frequency of sound is the rate at which the waves pass a given point, i.e. it is the number of oscillations (cycles) that occur each second of time. The amplitude, is the peak deviation of the function from zero. The simplest representation of a sound wave at a fixed point of the space is given by the sine wave, that is a sinusoid. Its most basic form as a function of time $t$ is:

$$
y(t)=A \sin (2 \pi \nu t+\phi)=A \sin (\omega t+\phi)
$$

where: $A$ is the amplitude of the wave, $\nu$ is the fundamental frequency, $\omega=2 \pi \nu$ is the angular frequency, that is the rate of change of the function argument in units of radians per second, $\phi$ is the phase, and specifies (in radians) where in its cycle the oscillation is at $t=0$. If we fix our attention on sound waves generated by strings, the fundamental frequency $\nu$ is given by

$$
\nu=\frac{1}{2 L} \sqrt{\frac{T}{\mu}},
$$


where $L$ is the length of the string, $T$ is its tension, and $\mu$ is its density. Sine waves are very important in music, they may be considered the expression of the vibration with a single frequency. Generally the motion of the source of sound is more complicated and the produced sound waves are a sum of several sine waves with different frequencies, all occurring at the same moment. These frequencies are all multiples of the fundamental one $\nu$. A more datelined discussion of these topics is given in [4].

\section{Construction of a musical scale}

Around 500 B.C. Pythagoras studied the musical scale connecting the sounds with the length of the string that produces them, by using a simple mechanical tool called monochord. Pythagoras determined a seven notes scale using just three ratios between notes: 2:1 called octave (because it is the eighth note in a seven-note scale), 3:2 called a perfect fifth (because it is the position of the fifth note in a modern scale), 4:3 called a perfect fourth (because it is the position of the fourth note in a modern scale). After he created the second note by increasing the fifth of another fifth (9:4), and by using the 1/2 rule in order to remain in the same octave, hence the second note corresponds to 9:8. Similarly:

- the ratio for the third was $9 / 8 \times 9 / 8=81 / 64$;

- the ratio for the sixth was $9 / 8 \times 3 / 2=27 / 16$;

- the ratio for the seventh was $3 / 2 \times 9 / 8 \times 9 / 8=243 / 128$.

Some of these ratios were corrected in the so called natural just scale, that is a scale in which the frequencies of notes are related by ratios of small whole numbers (just intonation). This tuning system of the later ancient Greek modes was codified by Claudius Ptolemy (about 85-165 AD) and developed by Gioseffo Zarlino (Istitutioni Harmoniche 1558). The corrections made on the Pythagorean scale are the following: 81:64 was corrected in 5:4, 27:16 in 5:3 and 243:128 in 15:8 (see the table below).

\begin{tabular}{|c|cccccccc|}
\hline note & $\mathrm{C}$ & $\mathrm{D}$ & $\mathrm{E}$ & $\mathrm{F}$ & $\mathrm{G}$ & $\mathrm{A}$ & $\mathrm{B}$ & $\mathrm{C}$ \\
\hline Pythagorean & 1 & $9 / 8$ & $81 / 64$ & $4 / 3$ & $3 / 2$ & $27 / 16$ & $243 / 128$ & $2 / 1$ \\
\hline natural & 1 & $9 / 8$ & $5 / 4$ & $4 / 3$ & $3 / 2$ & $5 / 3$ & $15 / 8$ & $2 / 1$ \\
\hline
\end{tabular}

Nowadays the most used musical scale in Western countries is the equal temperament scale, which divides the octave into 12 parts, all of which are equal on a logarithmic scale, that we describe in the last part of the paper. It is usually tuned relative to a standard pich of $440 \mathrm{~Hz}$, called A440. 


\subsection{The tempered scale}

As shown, the vibration of a cord is the result of the overlapping of the frequencies $f, 2 f, 3 f, \ldots$, called harmonics of the fundamental frequency $f$. Some experiments, performed with a monochord, highlight that doubling the frequencies of consecutive sounds gives a especially nice effect when they overlap. To build and tune instruments with fixed sounds (harpsichord, piano, ... ) the tempered scale is used. Moreover, this scale offers the possibility of modular compositions, i.e. has the feature to move from a tonality to another.

In order to construct musical scales, that are nice to hear, it is necessary to follow certain rules: if a scale has a frequency $f$ then it should have also the frequency $2 f$ and then the frequency $f / 2$. The main issue is that the sound sequences give a melody portable on our scale, in other words the melody should be able to be reproduced by starting from a sound of any frequency. The melody is characterized by the ratios of the frequencies of consecutive sounds for psychoacoustic reasons. Since we wish to play a melody from different frequencies, these ratios should be assigned constant. Let

$$
\underset{440 \mathrm{~Hz}}{f_{0}}<f_{1}<f_{2}<\cdots<f_{m}=\underset{880 \mathrm{~Hz}}{2 f_{0}}
$$

be the frequencies of the sounds in the octave $[f, 2 f]$, which is subdivided into $m$ intervals. Now, let us shift the melody (1) right an interval, i.e. by starting from $f_{1}$ and by ending at $f_{m+1}$. We obtain the following two melodies:

$$
\begin{gathered}
f_{0}<f_{1}<f_{2}<\cdots<f_{m} \\
f_{1}<f_{2}<f_{2}<\cdots<f_{m+1} .
\end{gathered}
$$

If the initial melody has not been altered, then it holds that:

$$
\frac{f_{1}}{f_{0}}=\frac{f_{2}}{f_{1}}, \quad \frac{f_{2}}{f_{1}}=\frac{f_{3}}{f_{2}}, \quad \cdots, \quad \frac{f_{m}}{f_{m-1}}=\frac{f_{m+1}}{f_{m}},
$$

and so

$$
\frac{f_{1}}{f_{0}}=\frac{f_{2}}{f_{1}}=\frac{f_{3}}{f_{2}}=\cdots=\frac{f_{m+1}}{f_{m}}=q .
$$

Hence the sequence $f_{0}, \ldots, f_{m}$ is a geometric progression of ratio $q=\sqrt[m]{2}$, i.e.:

$$
f_{k}=q^{k} f_{0}, \quad \text { for } \quad k=0,1, \ldots, m, \quad f_{m}=q^{m} f_{0}=2 f_{0} .
$$

The problem is to find an integer number $m$ of intervals that divides the octave. By considering the sequence of the logarithms to the base 2 of the frequencies:

$$
\log _{2} f_{0}, \quad \log _{2} f_{1}, \quad \log _{2} f_{2}, \cdots, \log _{2} f_{m}
$$


and observing that

$\log _{2} f_{k}=\log _{2}\left(f_{0} \cdot q^{k}\right)=\log _{2} f_{0}+k \log _{2} q=\log _{2} f_{0}+\frac{k}{m}, \quad$ for $\quad k=0,1, \ldots, m$,

the octave is transported in an unitary interval, and the sequence of the frequencies becomes an arithmetic progression of ratio $\lambda=\log _{2} q=\frac{1}{m}$. By these remarks, if the scale contains the frequency $f$ it has also the $3 f$ value and therefore the frequency $3 / 2 f$, which is contained in the octave $[f, 2 f]$. By assuming that such frequency occupies the $k$-th place in the scale, it results

$$
f_{k}=\frac{3}{2} f_{0} \quad \text { and } \quad \log _{2} f_{k}=\log _{2} \frac{3}{2}+\log _{2} f_{0} .
$$

Then the problem is to solve the equation

$$
\log _{2} \frac{3}{2}=\frac{k}{m}
$$

where $k, m$ are integer numbers. We notice that $\log _{2} \frac{3}{2}$ is an irrational number ${ }^{1}$ then equation (3) admits no integer solutions. Then the problem is reduced to compute an approximate solution of the equation (3), i.e. two integers $k, m$ such that the difference

$$
\operatorname{err}(k, m)=\left|\log _{2} \frac{3}{2}-\frac{k}{m}\right|
$$

is small. Moreover, we are interested to the minimal solution, i.e. a solution with $m$ as small as possible. A more rigorous mathematical formulation of this problem is the following.

Problem 1. Let be tol a given tolerance. Find a pair of integer numbers $(k, m)$ such that:

$$
m=\min \left\{\bar{m} \in \mathbb{N}|\exists \bar{k} \in \mathbb{N}:| \frac{\bar{k}}{\bar{m}}-\log _{2} \frac{3}{2} \mid<\text { tol }\right\}
$$

Notice that, once $m$ has been fixed, the value of $k$ that minimizes the difference $\operatorname{err}(k, m)$ is given by

$$
k=\operatorname{round}\left(m \cdot \log _{2} \frac{3}{2}\right),
$$

where the symbol $\operatorname{round}(x)$ gives the rounding to a integer number of $x$. Then, an algorithm to solve the Problem 1 is the following.

\footnotetext{
${ }^{1} \mathrm{~A}$ proof by contradiction is the following. Suppose that $\log _{2} \frac{3}{2}$ is a rational number, then there exist non-negative integers $k, m$ that solve equation (3). Taking the power to the base 2 of both sides yields

$$
\frac{3}{2}=2^{\frac{k}{m}} \quad \text { and so } \quad\left(\frac{3}{2}\right)^{m}=2^{k} \quad \Leftrightarrow \quad 3^{m}=2^{m+k} .
$$

However $3^{m}$ is odd while $2^{m+k}$ is even, therefore $3^{m}=2^{m+k}$ is impossible. Hence the first statement must be false and $\log _{2} \frac{3}{2}$ is irrational.
} 


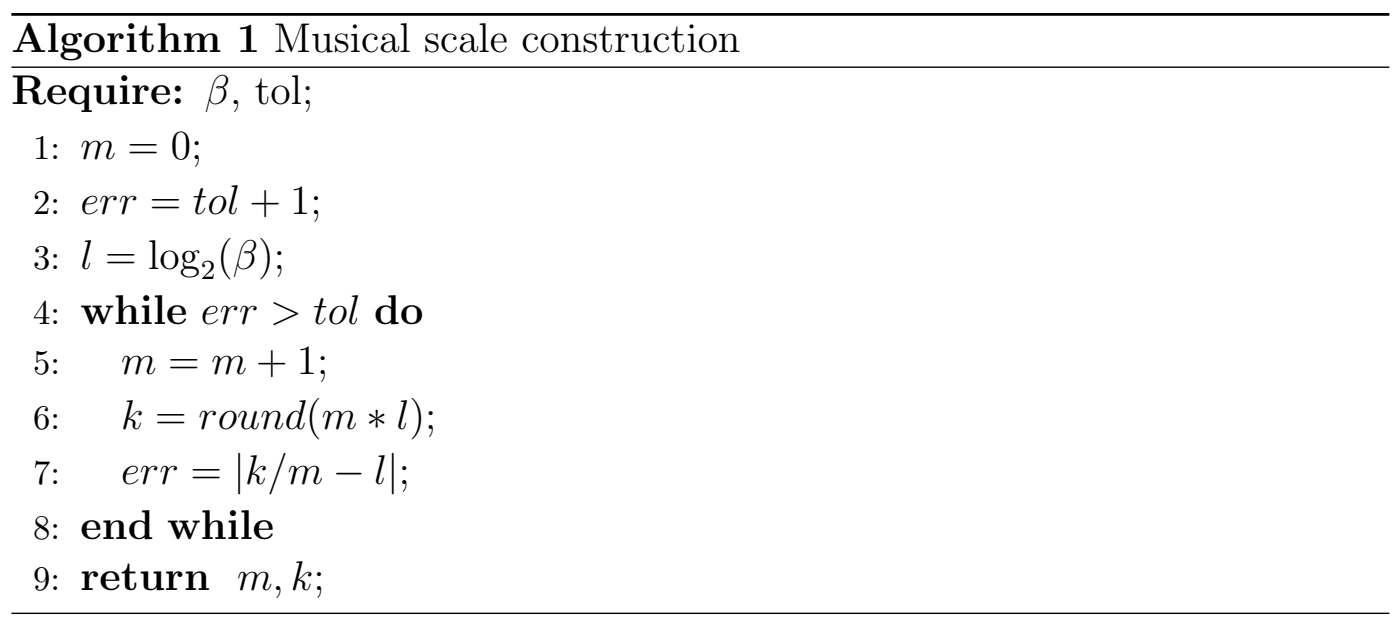

For psychoacoustic reasons the sounds with frequency less the $1 \mathrm{~Hz}$ are imperceptible to the human ear. Then, according to this remark, the tolerance tol should be chosen to be equal to ${ }^{2}$ 0.004. By using Algorithm 1, with $\beta=3 / 2$ and $t o l=0.004$, we get the pair:

$$
(k, m)=(7,12),
$$

i.e. the fraction of integers $\frac{7}{12}$ is an acceptable approximation in the discussed sense. Once the value of $m$ has been found, it is necessary to approximate in a satisfactory manner also the values related to te other frequencies: the third $5 / 4$, the fourth $4 / 3$, the sixth $5 / 3$, the second $9 / 8$ and the seventh $15 / 8$. By a mathematical point of view, it means that, given $m$, the problem is to find an approximate solution of:

$$
\log _{2} \beta_{i}=\frac{k_{i}}{m} \quad \text { with } \quad m=12, k_{i} \in \mathbb{N},
$$

where $\beta_{i}=5 / 4,4 / 3,5 / 3,9 / 8,15 / 8$. This can be obtained taking the values

$$
k_{i}=\operatorname{round}\left(m \cdot \log _{2} \beta_{i}\right) .
$$

In the following table, we report the computed values of $k_{i}$ and the committed error approximating $\log _{2} \beta_{i}$ with $\frac{k_{i}}{m}$.

\begin{tabular}{|c|c|c|c|c|c|}
\hline$\beta_{i}$ & $5 / 4$ & $4 / 3$ & $5 / 3$ & $9 / 8$ & $15 / 8$ \\
\hline $\log _{2} \beta_{i}$ & 0.3218 & 0.4150 & 0.7376 & 0.1699 & 0.9069 \\
\hline$\frac{k_{i}}{m}=\frac{\operatorname{round}\left(12 \log _{2} \beta_{i}\right)}{12}$ & $\frac{4}{12}$ & $\frac{5}{12}$ & $\frac{9}{12}$ & $\frac{2}{12}$ & $\frac{11}{12}$ \\
\hline $\operatorname{err}\left(k_{i}, m\right)=\left|\log _{2} \beta_{i}-\frac{k_{i}}{m}\right|$ & 0.0114 & 0.0016 & 0.0013 & 0.0033 & 0.0098 \\
\hline
\end{tabular}

\footnotetext{
${ }^{2}$ The value 0.004 corresponds to $1 \mathrm{~Hz}$ in the $\log$ scale.
} 
The constructed scale, subdivided into $m=12$ equal intervals, was introduced by Andreas Werckmeister in 17th century (Musicalische Temperatur, 1691) and used by J.S. Bach in its opera "The Well-Tempered Clavier".

Finally, observe that to have a better approximation of the solution of (3), we can get a smaller value of $t o l$, for instance $t o l=0.001$. In this case, by using Algorithm 1 with $\beta=3 / 2$ and $t o l=0.001$, we obtain the optimal integer pair

$$
(k, m)=(31,53)
$$

So the best subdivision has 53 intervals and the frequency of $\frac{3}{2} f$ is at the 31 -st place. It would be interesting ask to a composer to write a musical work by using 53 sounds in each octave. For sake of completeness we report in the following table the values computed with $m=53$ for the other main frequencies.

\begin{tabular}{|c|c|c|c|c|c|}
\hline$\beta_{i}$ & $5 / 4$ & $4 / 3$ & $5 / 3$ & $9 / 8$ & $15 / 8$ \\
\hline $\log _{2} \beta_{i}$ & 0.3218 & 0.4150 & 0.7376 & 0.1699 & 0.9069 \\
\hline$\frac{k_{i}}{m}=\frac{\operatorname{round}\left(53 \log _{2} \beta_{i}\right)}{53}$ & $\frac{17}{53}$ & $\frac{22}{53}$ & $\frac{39}{53}$ & $\frac{9}{53}$ & $\frac{48}{53}$ \\
\hline $\operatorname{err}\left(k_{i}, m\right)=\left|\log _{2} \beta_{i}-\frac{k_{i}}{m}\right|$ & 0.0012 & 0.0001 & 0.0011 & 0.0001 & 0.0012 \\
\hline
\end{tabular}

\section{Conclusions}

In this paper we recall our past teaching experiences in several departmental projects for introducing the high school students to nice mathematical applications. We worked to this aim also in a project, named Piano Lauree Scientifiche (PLS), for the Department of Mathematics and Applications (University of Naples Federico II). For an interdisciplinary laboratory tool we give a short algorithm for the construction of a temperate musical scale. This procedure can easily written in a programming language to naively explore the connections between Mathematics and Music.

\section{References}

[1] N. Vilenkine, G. Chilov, V. Ouspenki, J. Lioubitch, L. Chor, Quelques applications des Mathematiques, Ed Mir Moscou 1975.

[2] C. Taylor, Exploring Music, IOP Publishing Ltd Bristol 1992

[3] J. G. Roederer, Introduction to the Physics and the Psychophysics of Music, Springer Verlag, Heidelberg 1979. 
[4] A.H. Benade, Fundamentals of musical acoustics, Oxford Univ. Press, London, 1976

[5] T.D. Rossing, The Science of Sound, Addison-Wesley, London 1982

[6] J. Fauvel, Music and Mathematics from Pythagoras to Fractals, R. Flood \& R. Wilson eds.,Oxford University Press 2003.

Received: May 9, 2014 